\title{
Snapin is Critical for Presynaptic Homeostatic Plasticity
}

\author{
Dion K. Dickman, Amy Tong, and Graeme W. Davis \\ Department of Biochemistry and Biophysics, University of California, San Francisco, San Francisco, California 94158
}

The molecular mechanisms underlying the homeostatic modulation of presynaptic neurotransmitter release are largely unknown. We have previously used an electrophysiology-based forward genetic screen to assess the function of $>400$ neuronally expressed genes for a role in the homeostatic control of synaptic transmission at the neuromuscular junction of Drosophila melanogaster. This screen identified a critical function for $d y s$ indin, a gene linked to schizophrenia in humans (Dickman and Davis, 2009). Biochemical studies in other systems have shown that Snapin interacts with Dysbindin, prompting us to test whether Snapin might be involved in the mechanisms of synaptic homeostasis. Here, we demonstrate that loss of snapin blocks the homeostatic modulation of presynaptic vesicle release following inhibition of postsynaptic glutamate receptors. This is true for both the rapid induction of synaptic homeostasis induced by pharmacological inhibition of postsynaptic glutamate receptors, and the long-term expression of synaptic homeostasis induced by the genetic deletion of the muscle-specific GluRIIA glutamate receptor subunit. Loss of snapin does not alter baseline synaptic transmission, synapse morphology, synapse growth, or the number or density of active zones, indicating that the block of synaptic homeostasis is not a secondary consequence of impaired synapse development. Additional genetic evidence suggests that snapin functions in concert with dysbindin to modulate vesicle release and possibly homeostatic plasticity. Finally, we provide genetic evidence that the interaction of Snapin with SNAP25, a component of the SNARE complex, is also involved in synaptic homeostasis.

\section{Introduction}

Stable yet flexible neural function is essential for the robust and persistent function of the nervous system. It is increasingly apparent that homeostatic signaling systems, potentially functioning at the level of individual presynaptic and postsynaptic cells, have a potent role in stabilizing neural function. For example, homeostatic signaling has been implicated in the regulation of ion channel abundance while maintaining intrinsic excitability (Marder and Goaillard, 2006; Bergquist et al., 2010; Turrigiano, 2011). Homeostatic signaling has also been demonstrated in the control of synaptic transmission through regulation of postsynaptic neurotransmitter receptor abundance (Turrigiano and Nelson, 2004; Turrigiano, 2008; Pozo and Goda, 2010) and presynaptic neurotransmitter release (Davis, 2006; Jakawich et al., 2010). Despite widespread implication of homeostatic signaling in the control of neural function, very little is understood about how homeostatic signaling systems are designed and im-

Received 0ct. 28, 2011; revised May 6, 2012; accepted May 11, 2012.

Author contributions: D.K.D. and G.W.D. designed research; D.K.D. performed research; D.K.D. and A.T. contributed unpublished reagents/analytic tools; D.K.D. analyzed data; D.K.D. and G.W.D. wrote the paper.

This work was supported by a postdoctoral fellowship from the Jane Coffin Childs Cancer Memorial Fund and an NIH Pathway to Independence Award (K99MH92351) from NIMH (to D.K.D.), and by an NIH grant to G.W.D. (NS39313). We thank David Deitcher, Esteban Dell'Angelica, Jill Wildonger, and Yuh Nung Jan for providing Drosophila stocks. We also acknowledge the Vienna Drosophila Research Consortium and the Bloomington Stock Center for providing Drosophila stocks. We thank C. Andrew Frank for providing the unpublished GluRIII RNAi line. We thank Martin Muller, Meg Younger, and D. J. Braiser for comments on an earlier version of the manuscript.

The authors declare no competing financial interests.

Correspondence should be addressed to Graeme W. Davis, Department of Biochemistry and Biophysics, 1550 Fourth Street, Rock Hall Fourth Floor North, University of California, San Francisco, San Francisco, California 94158. E-mail: graeme.davis@ucsf.edu.

D. K. Dickman's present address University of Southern California, Department of Biology, Neurobiology Section, Los Angeles, CA 90089.

DOI:10.1523/JNEUROSCI.5465-11.2012

Copyright $\odot 2012$ the authors $\quad 0270-6474 / 12 / 328716-09 \$ 15.00 / 0$ plemented in cellular and molecular terms. Gene discovery through forward genetic screens in model organisms has become a means to identify the molecular basis of homeostatic signaling in the nervous system (Dickman and Davis, 2009).

The Drosophila neuromuscular junction (NMJ) has been established as a model system for dissecting the mechanisms underlying the homeostatic control of presynaptic neurotransmitter release (Davis and Goodman, 1998; Davis, 2006; Kim and Ryan, 2010). In brief, impaired postsynaptic neurotransmitter receptor function leads to a compensatory increase in presynaptic vesicle release that precisely offsets the postsynaptic perturbation and restores normal muscle excitation in the continued presence of the perturbation to postsynaptic receptors. The fact that muscle excitation is precisely restored to baseline values in the continued presence of a perturbation defines this process as homeostatic. This form of homeostatic signaling is conserved from fly to human (Davis, 2006), implying that the underlying mechanisms will also be conserved.

Here we demonstrate that Snapin is a critical component of the presynaptic machinery responsible for the homeostatic modulation of vesicle release at the Drosophila NMJ. Snapin is a ubiquitously expressed protein that is highly conserved from fly to human (Buxton et al., 2003). Snapin was initially identified as a binding partner to SNAP25, a core component of machinery necessary for SNARE-mediated vesicle fusion (Ilardi et al., 1999). However, the molecular function of Snapin, particularly in modulating neurotransmission, remains poorly understood (Vites et al., 2004). Recent studies examining snapin mutant mice demonstrate that loss of snapin disrupts synaptic vesicle fusion (Tian et al., 2005; Pan et al., 2009). Snapin is also implicated in the trafficking of endosomes and has diverse functions in other tissues outside the nervous system (Cai et al., 2010). Our data define a 
new role for Snapin in promoting the homeostatic modulation of vesicle release. This function can be separated from other actions of Snapin, including synapse stability and evoked vesicle release. Finally, Snapin functions in concert with Dysbindin and SNAP25 during synaptic homeostasis. dysbindin, a schizophrenia susceptibility gene in human, is essential for synaptic homeostasis (Dickman and Davis, 2009). These data provide a broader molecular context for considering a potential link between homeostatic signaling and neurological disease.

\section{Materials and Methods}

Fly stocks and genetics. All fly stocks were maintained at $22-25^{\circ} \mathrm{C}$ on normal food. The snapin and DAMT (CG9960) RNAi lines, as well as the UAS-dicer2 lines used in this study, were obtained from the Vienna Drosophila Research Center. We obtained the SNAP25 $5^{m \times 124}$ mutation (Vilinsky et al., 2002) from David Deitcher (Cornell University, Ithaca, NY) and the Dynein Intermediate Chain mutant, $\operatorname{dic}^{1229}$ (Zheng et al., 2008) from Jill Wildonger and Yuh Nung Jan (University of California, San Francisco, San Francisco, CA). We obtained blos $1^{E x 2}$ from Esteban Dell'Angelica (University of California, Los Angeles, Los Angeles, CA). Unless otherwise noted, all other fly lines were obtained from the Bloomington Drosophila Stock Center. Standard second- and thirdchromosome balancers and genetic strategies were used for all crosses and for maintaining mutant lines. In all experiments, unless specified otherwise, the $w^{1118}$ strain was used as the genetic background since this is the background in which transgenic flies are generated, and experiments were performed on female larvae.

Molecular biology. Drosophila snapin cDNA was obtained by amplifying the small, single open reading frame from genomic DNA by PCR and cloning directly in to the pENTR vector (Gateway Technology; Invitrogen). We engineered a CACC site in the forward primer for the subsequent Gateway reaction: forward primer for pUASt-snapin and pUASt-snapinvenus: 5 ' CACCATGGATTCGGACAGCACC 3'; reverse primer for pUASt-snapin: 5' TTAGGATATGTTGGTGCTGAG 3'; reverse primer for pUASt-snapin-venus: 5' GGATATGTTGGTGCTGAGC 3'. All final constructs were sequenced to ensure there were no mutations. The snapin cDNA was then cloned into proper destination vectors obtained from the Drosophila Gateway Vector Collection (Carnegie Institution). Transgenic lines were generated and mapped using standard methods.

Electrophysiology. Recordings were made in modified HL3 saline at specified calcium concentrations (see figures and text) with the following components (and concentrations): $\mathrm{NaCl}(70 \mathrm{~mm}), \mathrm{KCl}(5 \mathrm{~mm}), \mathrm{MgCl}_{2}(10 \mathrm{~mm})$, $\mathrm{NaHCO}_{3}(10 \mathrm{~mm})$, Sucrose (115 mM), Trehalose (5 mm), HEPES pH 7.2 (5 $\mathrm{mm})$, and $\mathrm{CaCl}_{2}(0.5 \mathrm{~mm}$ unless specified otherwise). For acute pharmacological homeostatic challenge, semi-intact preparations, with the CNS, fat, and gut left intact, were perfused with Philanthatoxin-433 (PhTx; Sigma). PhTx was prepared as a stock solution (5 mM in water) and diluted in HL3 saline to $10 \mu \mathrm{m}$. Following an incubation of $10 \mathrm{~min}$, the preparations were rinsed and the dissection was completed. The cut motor axon was stimulated, and average excitatory junction potential (EJP), miniature EJP $(\mathrm{mEJP})$, and quantal content were calculated for each genotype. Muscle input resistance $\left(R_{\mathrm{in}}\right)$ was monitored at the beginning and end of each recording. Recordings were excluded if $R_{\text {in }}$ or $V_{\mathrm{m}}$ changed by $>10 \%$. Quantal content was determined for each recording by calculating the average EJP/ average mEJP and correcting for nonlinear summation (Martin, 1955). Quantal contents were calculated for each recording and then averaged across all NMJs for a given genotype. In no case did the correction for nonlinear summation alter the statistical significance of our data. Spontaneous and evoked EJPs were analyzed using MiniAnalysis (Synaptosoft), and data were analyzed using Microsoft Excel and GraphPad Prism. All comparisons were analyzed using both Student's $t$ test and either a one-way or two-way ANOVA including Bonferroni posttest. In all cases, the conclusions and statistical significance remained the same for both types of analysis. Corrections for nonlinear summation do not alter statistical significance of any comparisons.

Immunocytochemistry. Wandering third-instar larvae were dissected in ice-cold $0 \mathrm{Ca}^{2+} \mathrm{HL} 3$ and fixed in Bouin's fixative (Sigma-Aldrich) for 2 min. Larvae were washed briefly in PBST (PBS plus 0.5\% Triton) and incubated overnight at $4^{\circ} \mathrm{C}$ in primary antibody, washed the next day, and incubated in fluorescently conjugated secondary antibodies for $2 \mathrm{~h}$ at room temperature. The following antibodies were used: mouse antiBruchpilot (BRP) 1:100 (Kittel et al., 2006); rabbit anti-synaptotagmin 1:2000 (Littleton et al., 1993); rabbit anti-Dlg 1:4000 (Pielage et al., 2006); mouse anti-Flag M2 1:5000 (Sigma). Alexa-conjugated secondary antibodies and directly conjugated goat anti-HRP were used at 1:400 (Jackson Immunoresearch Laboratories, Invitrogen). Larval preparations were mounted in Vectashield (Vector) and imaged at room temperature using an Axiovert 200 (Zeiss) inverted microscope, a 100× Plan Apochromat objective (aperture 1.4), and a cooled CCD camera (Coolsnap HQ, Roper). Quantification of bouton and BRP number as well as BRP density was performed as described previously (Dickman and Davis, 2009). Intelligent Imaging Innovations software was used to capture, process, and analyze images.

Quantitative RT-PCR. Quantitative real-time (RT) PCR methods were performed as described previously (Bergquist et al., 2010). Briefly, primer probes specific for real-time PCR detection of Drosophila snapin, DAMT (CG9960), and ribosomal protein L32 (RpL32) were designed and developed by Applied Biosystems. The CNS or body wall musculature was removed from 25 third-instar larvae per sample (3-6 samples/genotype). Total RNA was isolated using a Qiagen RNeasy kit. A DNase digestion removed potential DNA contamination (RQ1 RNase-free DNase; Promega). Reverse transcription was performed (multiscribe reverse transcriptase; Applied Biosystems) using random hexamers and 1 $\mu \mathrm{g}$ total RNA. A no-RT control was performed for each sample. cDNA from the reverse transcription reaction was used as a template in a $10 \mu \mathrm{l}$ PCR (TaqMan Universal PCR Master Mix, no AmpErase UNG; Applied Biosystems). This $10 \mu \mathrm{l}$ reaction was done in triplicate. In addition, one $10 \mu \mathrm{l}$ no-RT reaction was used for each sample. The ABI Prism 7900 was used for all PCRs. Cycle threshold $\left(\mathrm{C}_{\mathrm{T}}\right)$ was determined by automated threshold analysis using SDS2.3 software according to the manufacturer's instructions (Applied Biosystems). Comparative snapin and DAMT levels (between control and knock-down mutant animals) were determined using the $\Delta \Delta \mathrm{C}_{\mathrm{T}}$ method (Applied Biosystems, according to included instructions). To determine whether the two amplification reactions had the same PCR efficiency, $\Delta \mathrm{C}_{\mathrm{T}}\left(\mathrm{C}_{\mathrm{T}}\right.$ of experimental gene $\mathrm{C}_{\mathrm{T}}$ of reference gene) values were determined across the serial dilutions and plotted against the log of the cDNA dilution. A slope close to zero indicates equivalent amplification efficiency. This was done and both snapin and DAMT had approximately equal amplification efficiencies compared with the endogenous control, RpL32.

\section{Results}

In Drosophila, the genetic locus that includes the snapin gene is quite complex. Several lines of evidence suggest that snapin expression is under dicistronic control, being coexpressed as a single transcript along with a separate open reading frame, CG9960 (Fig. 1A) (Wall et al., 2005). The CG9960 gene encodes a protein that is conserved from yeast to human: DNA adenine methyltransferase (DAMT; www.flybase.org). Currently, there are no available transposon insertions or other mutations that disrupt the snapin locus in Drosophila. However, we obtained two independent RNAi transgenes, one containing a sequence targeting the snapin open reading frame (ID 49288; $\operatorname{snapin}^{R N A i}$ ) and another targeting DAMT (ID 12574; DAMT ${ }^{R N A i}$; Fig. $1 A$ ). When we expressed these RNAi transgenes ubiquitously, both led to pupal lethality, indicating that transcription from this gene locus is necessary for viability. To determine levels of expression after knock down, we determined RNA expression using quantitative RTPCR (see Materials and Methods, above; Fig. $1 B$ ). Ubiquitous knock down using the snapin ${ }^{R N A i}$ transgene reduced both snapin and DAMT transcript levels by at least $63 \%$ in the nervous system and $70-80 \%$ in muscle. Thus, gene knock down is effective and sequences targeting the snapin gene also reduce DAMT expression, confirming the dicistronic nature of this gene locus. 

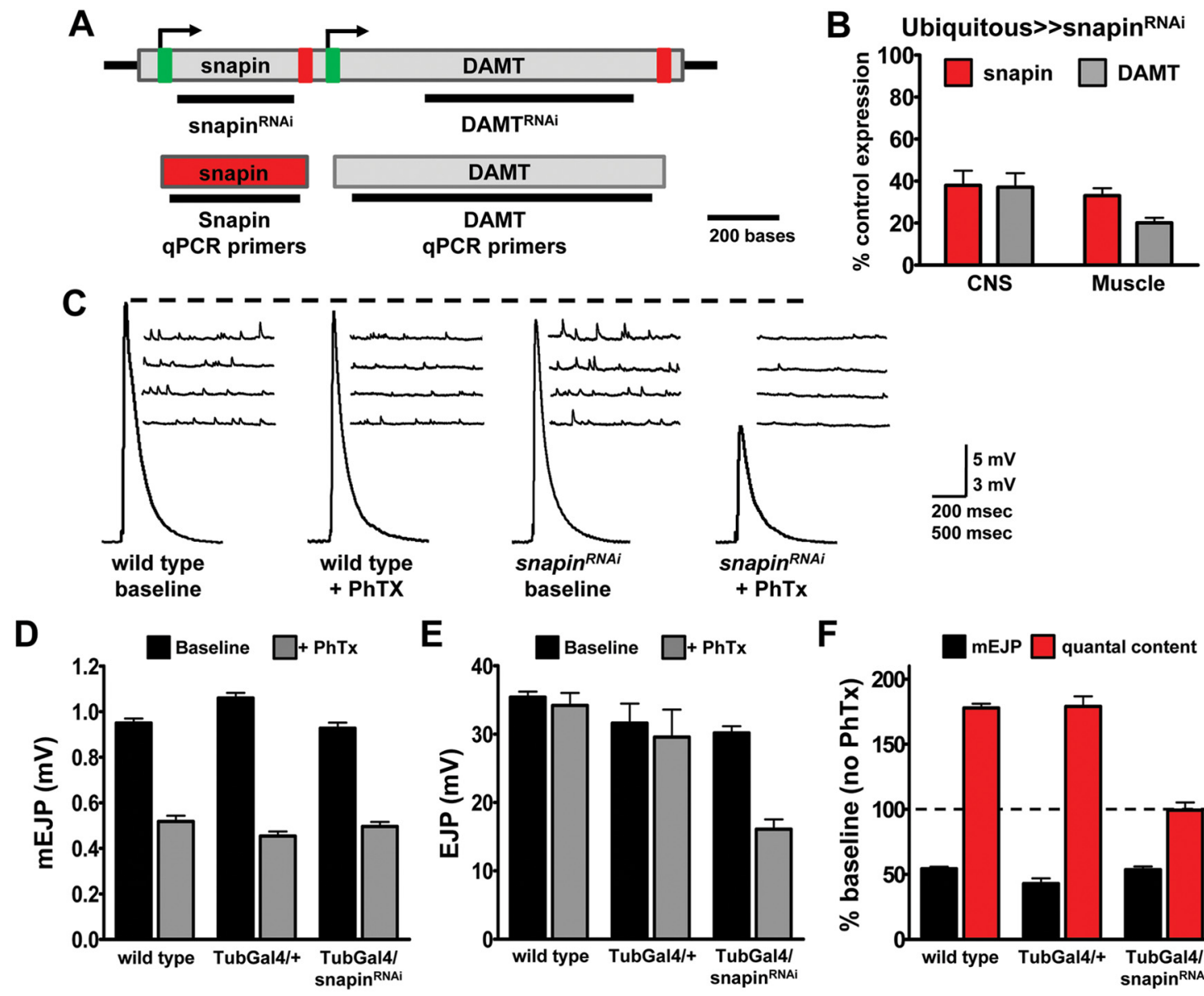

DAMT ${ }^{\text {RNAi }}$

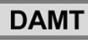

DAMT

PCR primers
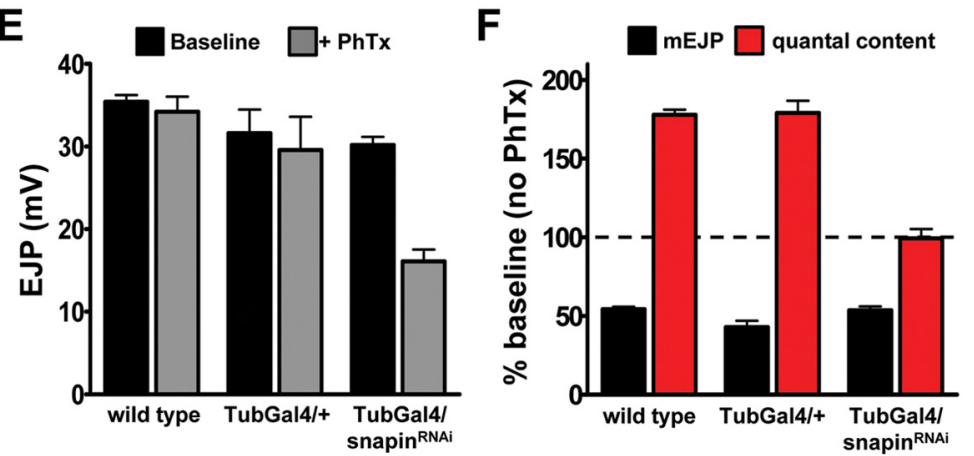

Figure 1. Ubiquitous knock down of the Drosophila snapin locus blocks synaptic homeostasis. A, Schematic of the dicistronic Drosophila snapin locus containing the snapin and damt (CG9960) open reading frames. Sequences contained in the snapin ${ }^{R N A i}$ or damt ${ }^{R N A i}$ transgenes as well as quantitative PCR (qPCR) primer regions are shown. $\boldsymbol{B}$, Ubiquitous knock down of snapin/damt (w; snapin ${ }^{\text {RAi }} / T u b u$ lin-GAL4) results in a large reduction in RNA expression of both genes by quantitative RT-PCR. D, E, Baseline electrophysiological recordings following PhTx application were performed and $\mathrm{mEJP}(\boldsymbol{D})$ and EJP $(\boldsymbol{E})$ values of wild-type $[w ;+;+; n=17 / 16(-/+\mathrm{PhTx})]$, driver alone ( $w ;$ Tubulin-GAL4/ $+; n=9 / 7)$, and snapin knock down ( $w ;$ snapin ${ }^{\text {RNAi } / T u b u l i n-G A L 4 ; n=}$ 12/11) are shown. $F$, Ubiquitous RNA knockdown of the snapin/DAMT locus blocks the homeostatic increase in quantal content following PhTx application ( $p<0.0001$ Student's $t$ test and ANOVA). Data are normalized to baseline values shown in $\boldsymbol{C}$ and $\boldsymbol{D}$. C, Representative traces for data in $\boldsymbol{F}$. Error bars are SEM.

\section{Snapin is necessary for the rapid induction of synaptic} homeostasis

Knock-down of both snapin and DAMT expression does not cause larval lethality, allowing us to characterize the requirement of these genes for baseline synaptic transmission and homeostatic plasticity at the larval NMJ. Homeostatic plasticity at the Drosophila NMJ is induced following disruption of postsynaptic glutamate receptor function (Petersen et al., 1997; Frank et al., 2006). Impaired glutamate receptor sensitivity causes a decrease in miniature excitatory junctional potential (mEJP) amplitude recorded from muscle. Reduced postsynaptic mEJP amplitudes in turn induce an increase in presynaptic neurotransmitter release that precisely offsets the change in $\mathrm{mEJP}$ amplitude, restoring action potential-induced muscle depolarization (excitatory junctional potential (EJP) amplitudes) to baseline values. Because baseline EJP amplitudes are restored to baseline levels in the continued presence of the perturbation (decreased glutamate receptor sensitivity), this form of presynaptic modulation is referred to as presynaptic homeostatic plasticity (Davis, 2006).

There appear to be two phases of presynaptic homeostatic plasticity. First, homeostatic modulation of presynaptic release can be rapidly induced (seconds to minutes) following application of the postsynaptic glutamate receptor antagonist PhTx. This form of homeostatic modulation is independent of new transcription and translation (Frank et al., 2006). Second, homeostatic plasticity can be induced following loss of the postsynaptic glutamate receptor subunit GluRIIA or by reduced expression of the postsynaptic glutamate receptor subunit GluRIII (Petersen et al., 1997; Marrus et al., 2004). These postsynaptic perturbations persist throughout larval life and loss of the transcription factor gooseberry specifically disrupts homeostatic plasticity in these genetic backgrounds, suggesting that the maintenance of homeostatic plasticity will require new gene transcription (Marie et al., 2010). These forms of homeostatic plasticity are evolutionarily conserved from fly to human and similar forms of homeostatic plasticity may exist in the vertebrate CNS (Pozo and Goda, 2010).

Here we demonstrate that simultaneous knock down of snapin and damt has negligible effects on baseline synaptic transmission, but completely blocks presynaptic homeostatic plasticity. Specifically, ubiquitous snapin knock down has little effect on baseline transmission, assayed by quantifying the average EJP amplitude and $\mathrm{mEJP}$ amplitude (see Materials and Methods, above). There is no statistically significant difference in average EJP amplitude when comparing knock-down animals to the heterozygous GAL4/+ control animals, although there is a minor, statistically significant $(p<$ 0.05 ) difference when comparing knock-down animals with wild-type (Fig. 1). The difference in EJP amplitudes comparing knock-down animals with wild-type cannot be accounted for by 

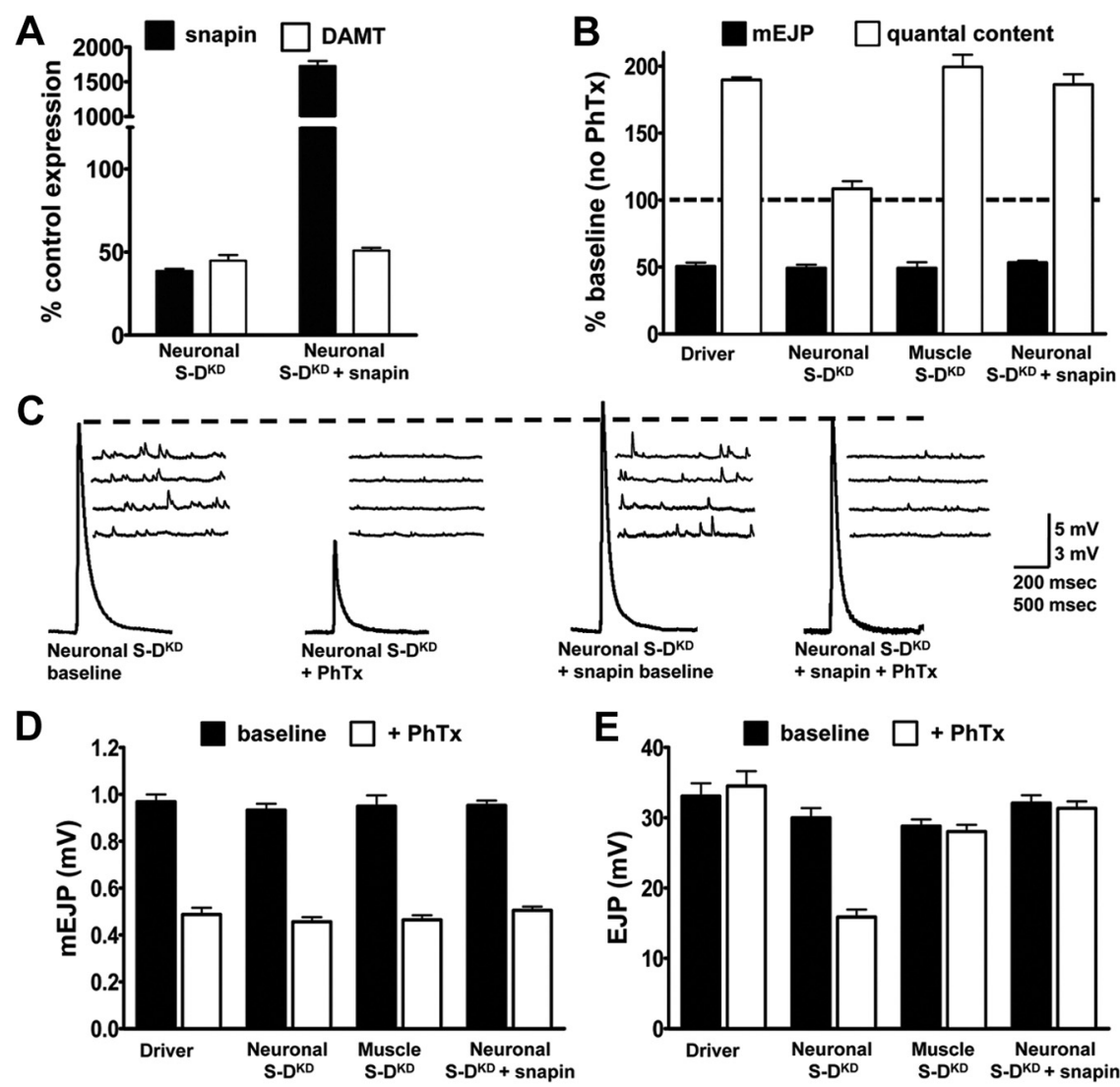

Figure 2. Snapin is required presynaptically for synaptic homeostasis. A, Quantitative RT-PCR of snapin and damt expression

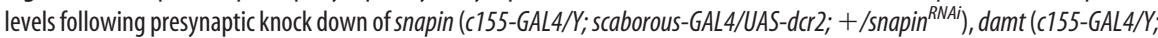
scaborous-GAL4/damt ${ }^{\text {RNAi }}$; UAS-dcr2/+), and damt combined with snapin overexpression (c155/Y; scaborous-GAL4/damt ${ }^{\text {RNAi; }}$; UAS-dcr2/UAS-snapin). $\boldsymbol{B}$, Presynaptic knock down of the snapin/damt locus using damt ${ }^{\text {RNAi }}$ block homeostatic compensation $(n=$ 22/14), while the driver alone (c155-GAL4/Y; scaborous-GAL4/+; UAS-dcr2/+; $n=8 / 9$ ) and postsynaptic knockdown ( $w$; damt $t^{\text {NAi }} /+;$ MHC-GAL4/UAS-dcr2; $\left.n=10 / 11\right)$ do not effect this process $(p<0.002$, Student's $t$ test and ANOVA). Synaptic homeostasis can be restored by coexpressing a snapin transgene after snapin/damt knock down $(n=12 / 14)$. $\boldsymbol{C}-\boldsymbol{E}$, Representative traces $(\boldsymbol{C}), \mathrm{mEJP}(\boldsymbol{D})$, and EJP $(\boldsymbol{E})$ values for data in $\boldsymbol{B}$. Error bars are SEM.

a change in average mEJP amplitude (Fig. 1C-E). The lack of effect on baseline synaptic transmission following snapin knock down is independently confirmed using a motoneuron-specific GAL4 driver (Fig. 2).

We then tested homeostatic plasticity. After incubation in PhTx to acutely inhibit postsynaptic glutamate receptor function, average mEJP amplitude is significantly reduced in both control and knock-down animals (Fig. $1 C-F$ ). In control animals, there is a robust homeostatic increase in presynaptic release that offsets the decrease in mEJP amplitude and restores EJP amplitudes to baseline values. However, in knock-down animals there is no homeostatic increase in presynaptic release and EJP amplitudes are significantly smaller than baseline. Thus, either snapin, damt, or both are necessary for the rapid induction of synaptic homeostasis at the Drosophila NMJ.

We next sought to determine which gene is critical for synaptic homeostasis, and in which tissue gene expression is required. Because Snapin is known to interact biochemically with Dysbindin, a gene previously shown to be required for synaptic homeostasis in this system (Dickman and Davis, 2009), we hypothesized that snapin may be a gene required for homeostatic plasticity. To test this hypothesis, we used the damt RNAi transgene to knock down expression of both genes. We then transgenically overexpressed snapin in either nerve or muscle to specifically restore snapin expression to the double knock-down animal. This experiment is possible because the damt RNAi line does not share sequences within the snapin transgene. We demonstrate the effectiveness of this approach by quantitative RT-PCR, showing that snapin can be stably expressed while damt expression remains knocked down (Fig. 2A).

We first demonstrate that expression of damt $t^{R N A i}$ (referred to as " $S-D^{K D}$," which stands for Snapin and DAMT knock-down) blocks synaptic homeostasis when expressed in the nervous system, but has no effect when expressed in muscle (Fig. 2B,C). Thus, expression of this locus is necessary in the nervous system for the expression of homeostatic plasticity. Next, we show that coexpression of snapin with damt ${ }^{R N A i}$ in the nervous system restores the expression of synaptic homeostasis (Fig. 2 B, C). From these data, we conclude that snapin is critically required in the nervous system for homeostatic plasticity. We further conclude that damt is not necessary for synaptic homeostasis or baseline neural function (Fig. $2 D, E)$. From this point on, we will refer to

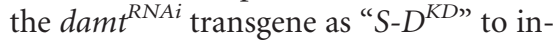
dicate that both transcripts are knocked down, and recognizing that snapin is the relevant transcript for synaptic homeostasis. The precise full genotype is always fully detailed in figure legends.

\section{Snapin is necessary for the maintenance} of synaptic homeostasis without altering NMJ morphology

Next, we determined whether snapin is required not only for the rapid induction of homeostatic plasticity but also for the long-term maintenance of synaptic homeostasis. To do so, we knocked-down glutamate receptor expression in muscle throughout development using an RNAi construct targeting the obligate glutamate receptor subunit GluRIII (see Materials and Methods, above) (Marrus et al., 2004; Qin et al., 2005). Reduced expression of GluRIII causes a reduction in $\mathrm{mEJP}$ amplitude and a homeostatic increase in presynaptic release (Fig. 3), similar to that observed in animals lacking the postsynaptic GluRIIA receptor subunit (Petersen et al., 1997). When we simultaneously reduce expression of GluRIII in muscle and snapin $\left(S-D^{K D}\right)$ in the nervous system, we find that homeostatic plasticity is completely blocked (Fig. 3). When considered with the demonstration that snapin is the relevant transcript for the rapid induction of synaptic homeostasis, we conclude that snapin expression is required for both the rapid induction and sustained expression of homeostatic plasticity.

Our previous work has demonstrated that homeostatic mutants do not necessarily show defects in synapse morphology, size, or active zone number or density (Frank et al., 2006; Dickman and Davis, 2009; Müller et al., 2011). Therefore, the underlying mechanisms of synaptic homeostasis can be dissociated from the mechanisms that drive normal morphological synapse growth (Dickman and Davis, 2009). This observation is confirmed in $S-D^{K D}$ animals, which have normal synapse morphology (Fig. $4 A-C$ ). Specifically, we quantified bouton number as 
well as the number and density of active zones using the active zone marker antiBRP (nc82), and found no significant difference comparing wild-type and $S-D^{K D}$ animals (Fig. 4C-E). Snapin was originally isolated as a synaptic vesicleassociated protein, and vertebrate studies have found that Snapin colocalizes with synaptic vesicle markers (Ilardi et al., 1999; Pan et al., 2009). We have confirmed this observation at the NMJ using a flag-tagged snapin transgene, observing that Snapin traffics to a region of the presynaptic bouton that coincides with other synaptic vesicle markers (Fig. $4 F$ and data not shown).

\section{Snapin functions with Dysbindin during synaptic homeostasis}

Previous studies have provided evidence that Snapin interacts biochemically with the schizophrenia-susceptibility gene Dysbindin (Talbot et al., 2006; Cheli et al., 2010; Ghiani and Dell'angelica, 2011). We previously demonstrated that Drosophila dysbindin mutations block both the rapid induction and sustained expression of synaptic homeostasis at the NMJ (Dickman and Davis, 2009). Therefore, we pursued experiments to test the possibility that Snapin functions in concert with Dysbindin during homeostatic plasticity. To do so, we performed genetic interaction experiments. A deficiency that removes the entire snapin locus, when used in trans with a wild-type chromosome, has no effect on synaptic homeostasis (Fig. $5 A-C)$. Likewise, a $d y$ sbindin mutation, as a heterozygote, does not alter synaptic homeostasis. However, the double-heterozygous condition of both alleles shows significantly less homeostatic compensation compared with either heterozygous mutant alone (double-heterozygous mutant $=135 \pm 5.63 \%$ compensation compared with wild-type $=178 \pm 7.31 \%$ compensation; $p<$ 0.01 , Student's $t$ test; Fig. $5 A-C$ ). One caveat is that the snapin deficiency uncovers several genes in addition to snapin, including synaptotagmin-1. Therefore, two additional experiments were performed to control for the possible involvement of synaptotagmin-1 or other genes contained in the deficiency. First, we examined double-heterozygous mutants for dysbindin and synaptotagmin (DiAntonio et al., 1993) and found that homeostatic compensation is normal ( $p<0.05$; Fig. $5 D$, Table 1$)$. This rules out an interaction between synaptotagmin and dysbindin, but does not control for possible interactions with one of the other genes contained in the deficiency (in addition to snapin). Therefore, we examined animals that carried heterozygous mutations in dysbindin and the snapin deficiency and then overexpressed snapin in motoneurons in this background. Synaptic homeostasis was restored toward control levels $(p>0.05$, Student's $t$ test compared with double heterozygote and ANOVA compared with all genotypes; Fig. 5C). Although homeostatic compensation remains reduced compared with $d y s b i n d i n /+$ animals, compensation is statistically significantly improved compared with the double-heterozygous mutant. These data indicate that loss of snapin expression is important for the observed genetic interaction with dysbindin.

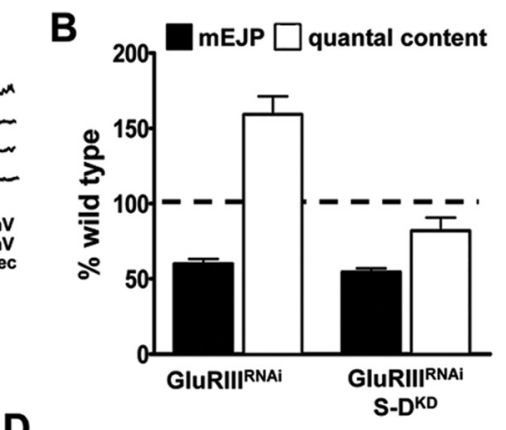

D
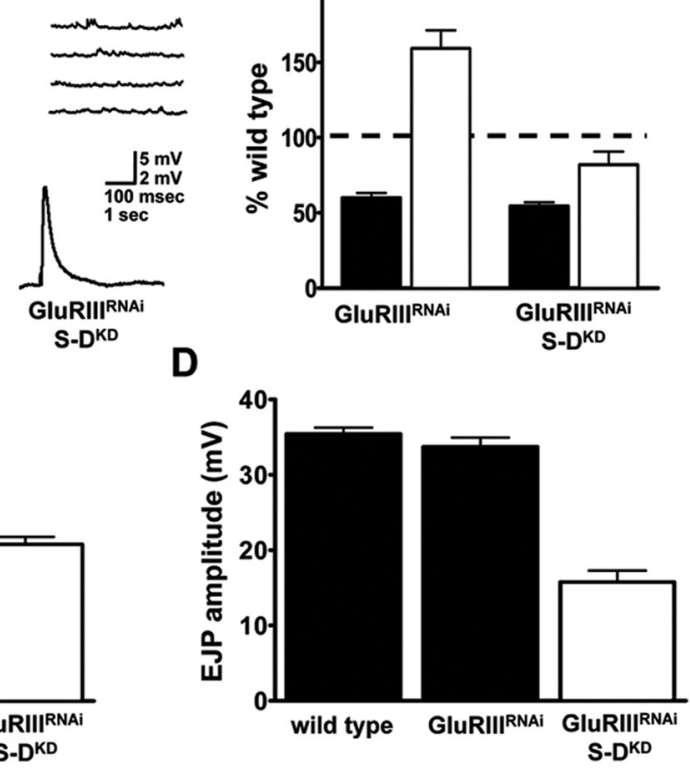

Figure 3. Snapin is required for the sustained expression of synaptic homeostasis. $\boldsymbol{A}$, Postsynaptic knock down of the glutamate receptor subunit GluRIII reduces mEJP amplitude throughout development, leading to a compensatory increase in presynaptic release to restore normal postsynaptic excitation. This process is blocked when snapin is also knocked down. Representative traces

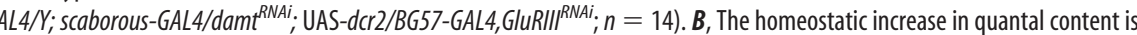
blocked when postsynaptic glutamate receptor function is reduced throughout development ( $p<0.018$ Student's $t$ test). C, $\boldsymbol{D}$, $\mathrm{mEJP}(\boldsymbol{C})$ and EJP $(\boldsymbol{D})$ values for normalized data in $(\boldsymbol{B})$. Error bars are SEM.

We also examined additional aspects of baseline synaptic transmission in further detail. We find that snapin knock down $\left(S-D^{K D}\right)$ does not alter the calcium cooperativity of release when transmission is assessed across a range of extracellular calcium concentrations. There is a minor reduction in baseline transmission across all calcium conditions assayed (Fig. 5E). Interestingly, this differs from dysbindin mutants when analyzing baseline transmission over a range of extracellular calcium concentrations (Dickman and Davis, 2009). Animals lacking dysbindin with simultaneous snapin knock down $\left(S-D^{K D}\right)$ show impaired synaptic transmission at lower extracellular calcium concentrations, but do not differ from $d y s b i n d i n$ mutants alone (Fig. 5F). Thus, although Dysbindin and Snapin both participate in homeostatic plasticity and may function together in this process, Dysbindin has a separable activity that alters the apparent cooperativity of presynaptic release.

We next determined the extent to which Snapin functions with Dysbindin to promote synaptic transmission. We have previously reported that overexpression of $d y$ sbindin in an otherwise wild-type background leads to potentiation of baseline release (Fig. 6A,B) (Dickman and Davis, 2009). Hence, we asked whether snapin overexpression also potentiates baseline synaptic vesicle release. Overexpression of snapin in the nervous system does not potentiate synaptic transmission (Fig. $6 A, B$ ). Interestingly, however, when dysbindin is overexpressed in the $S-D^{K D}$ background, dysbindin fails to potentiate release, indicating that normal snapin levels are required for the ability of $d y s b i n d i n$ to potentiate release. It is possible that DAMT mediates this effect, since it is also knocked down in this experiment. However, the reported biochemical interaction of Dysbindin and Snapin, and a genetic interaction during synaptic homeostasis, points strongly to loss of snapin being causal. Our results are consistent with the conclusion that Snapin functions with Dysbindin to influence 
A

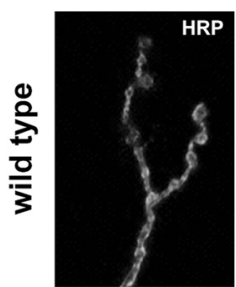

B

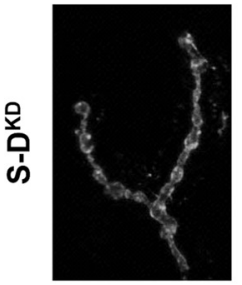

C

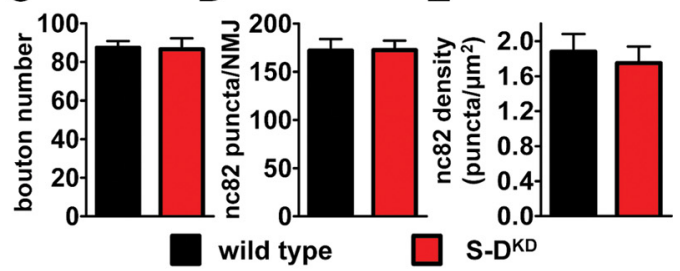

F
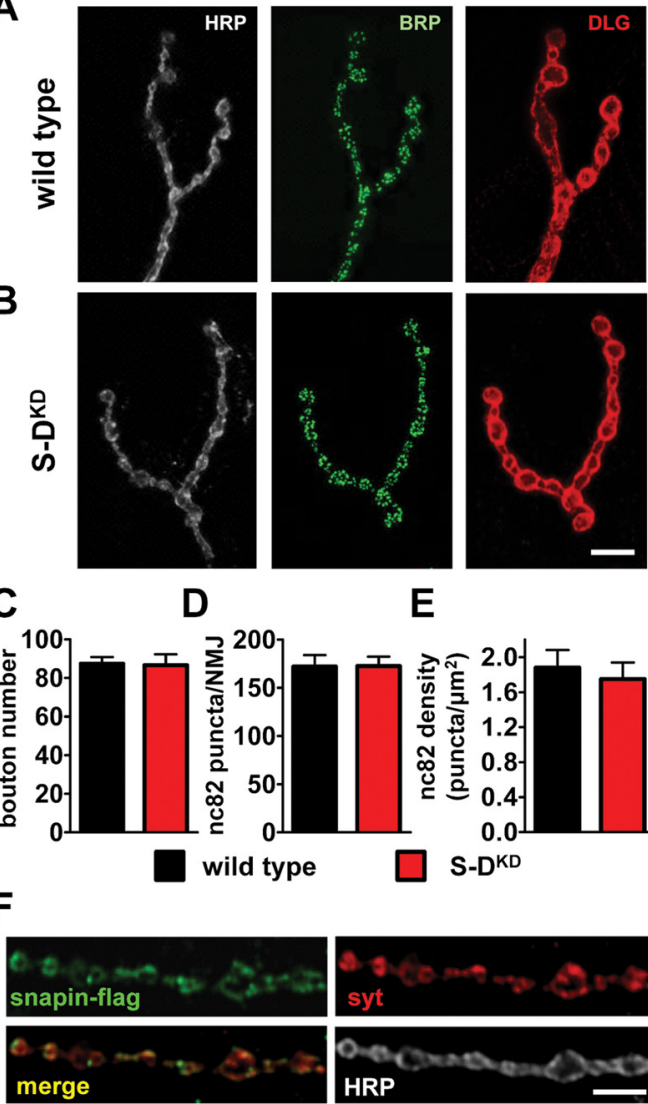

E

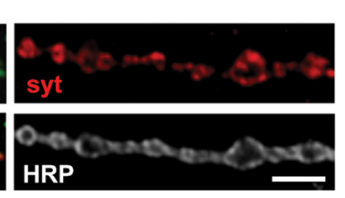

Figure 4. Snapin colocalizes with synaptic vesicle markers and displays normal synapse morphology and active zone number. $\boldsymbol{A}, \boldsymbol{B}$, Normal synapse morphology for $S-D^{K D}$ mutants. Representative images of the presynaptic membrane marker horseradish peroxidase (HRP; white), active zone marker Bruchpilot (BRP; green), and postsynaptic density marker Discs large (DLG; red) are shown. Scale bar, $10 \mu \mathrm{m}$. $\boldsymbol{C}-\boldsymbol{E}$, Quantification of bouton number (C), active zone number $(\boldsymbol{D})$, and active zone density $(\boldsymbol{E})$ in $S-D^{K D}$ mutants showing no significant difference in these parameters ( $p>0.05$, Student's $t$ test). Error bars are SEM. $\boldsymbol{F}$, Flag-tagged Snapin colocalizes with the synaptic vesicle marker Synaptotagmin (syt) near synaptic vesicle pools when expressed presynaptically (c155-GAL4; UAS-snapin-flag/+). Scale bar, $3 \mu \mathrm{m}$.

evoked neurotransmitter release. It is possible that Snapin functions downstream of Dysbindin. Alternatively, Snapin may function as a cofactor that is necessary for the full functionality of Dysbindin, consistent with data indicating that both Snapin and Dysbindin are part of the multiprotein subunit BLOC-1 complex. This latter possibility is supported by the observation that overexpression of Snapin alone is without effect on baseline synaptic transmission.

\section{Snapin genetically interacts with SNAP25 during synaptic homeostasis}

Snapin has been suggested to have two distinct and independent functions in neurons. The first function, through a biochemical interaction with the $t$-snare SNAP25, is to synchronize calciumdependent synaptic vesicle release (Ilardi et al., 1999; Pan et al., 2009). A second function, through a separate biochemical interaction with Dynein Intermediate Chain (DIC), is to promote retrograde trafficking of late endosomes from synaptic terminals to the soma (Cai et al., 2010). To test which of these functions may be relevant to Snapin's role in promoting synaptic homeostasis, we acquired a loss-of-function mutation in Drosophila $d y$ nein intermediate chain ( dic $^{1229}$ ) (Zheng et al., 2008) and tested for a genetic interaction with the snapin deficiency. However, we did not uncover a genetic interaction when $\operatorname{dic}^{1229}$ and snapin ${ }^{D F}$ were placed in trans as a double heterozygote $(p>0.05$, Student's $t$ test and ANOVA; Fig. 7A). In contrast, we observed a strong genetic interaction when a snap25-null mutation is placed in trans to the snapin deficiency in a double-heterozygous condition $(p<0.001$, Student's $t$ test and ANOVA; Fig. $7 B)$. Importantly, to control for the other genes removed in the snapin deficiency, expression of snapin in motor neurons restored homeostatic compensation in the double-heterozygous snapin ${ }^{D f} /+$; snap25/+ mutant (Fig. $7 B$ ). Although the lack of a genetic interaction with DIC is not definitive, the fact that this genetic interaction failed while there was a strong interaction with snap25 suggests that the biochemical interactions with SNAP25 are likely to be the most relevant to synaptic homeostasis at the Drosophila NMJ, while the proposed function of Snapin during endosomal trafficking may not be directly relevant to homeostatic plasticity. Of course, additional experimentation will be necessary to further explore this possibility.

Finally, we explored the possibility that Snapin functions with the BLOC-1 complex during homeostatic synaptic plasticity. The BLOC-1 complex is a multi-subunit protein complex implicated in endosomal trafficking and shown to be relevant to the cellular activity of both Snapin and Dysbindin. Snapin and Dysbindin are stoichiometric components of the BLOC-1 complex. To gain insight into the function of the Bloc-1 complex, we obtained a mutation a Drosophila BLOC-1 complex subunit, Blos1 (Cheli et al., 2010). This mutation is the only one for a BLOC-1 complex gene, outside of snapin and $d y$ sbindin, that has been molecularly characterized. Here we demonstrate that a null mutation in Blos 1 exhibits normal homeostatic compensation following application of PhTx (Fig. 7B), indicating the Blos1 protein is not involved in homeostatic plasticity. Although it has been hypothesized that components of the BLOC-1 complex necessarily functions together as a unit, snapin mutant mice have phenotypes that are not shared by other mutants of the BLOC-1 complex (Pan et al., 2009; Cai et al., 2010; Ghiani and Dell'angelica, 2011). Ultimately, it remains to be determined whether other members of the BLOC-1 complex will participate in the mechanisms of homeostatic plasticity. This represents a series of studies that are beyond the scope of our current manuscript.

\section{Discussion}

Here we provide evidence that Snapin promotes the homeostatic modulation of presynaptic neurotransmitter release, functioning presynaptically in concert with Dysbindin and SNAP25. It is important to emphasize that we are not examining snapin-null mutations and, therefore, we cannot make direct comparisons to the synaptic transmission phenotypes observed in snapin mutant mice. However, despite the limitation of assaying only the loss of snapin, we are able to conclude that reduced levels of snapin expression lead to the block in homeostatic plasticity without dramatically altering baseline transmission. We conclude that the mechanisms of synaptic homeostasis are particularly sensitive to the levels of Snapin, regardless of any additional function Snapin may have in evoked synaptic vesicle fusion.

We also provide several lines of evidence that snapin functions with the schizophrenia susceptibility gene $d y s$ bindin, most likely within motoneurons, where both Snapin and Dysbindin have been demonstrated to be required for homeostatic plasticity (Dickman and Davis, 2009). In particular, we demonstrate that the ability of Dysbindin to potentiate synaptic transmission requires normal snapin expression. An interesting possibility, based 
purely on our genetic data, is that Snapin promotes signaling between cytosolic Dysbindin and SNAP25, influencing vesicle release either directly or indirectly. This raises the intriguing possibility that this could be an important site of molecular regulation underlying the homeostatic modulation of presynaptic vesicle release. Indeed, separate studies have reported that changes in both $d y$ sbindin and snapin expression alter SNAP25 levels in the nervous system (Numakawa et al., 2004; Lu et al., 2009). Future in vivo imaging experiments will be required to directly test this possibility. These are important but challenging experiments and beyond the scope of the present study.

\section{Snapin as a homeostatic modulator of SNARE-mediated fusion}

The molecular function of Snapin remains poorly understood in the nervous system and in other tissues. snapin is highly conserved throughout evolution from invertebrates to rodents and human (Falcón-Pérez et al., 2002; Cheli and Dell'Angelica, 2010; Cheli et al., 2010). snapin is ubiquitously expressed in these organisms and does not seem to be enriched within the nervous system. In addition, Snapin protein is present at low levels within neurons compared with other SNARE complex proteins including SNAP25 (Vites et al., 2004). These observations have led some groups to question whether Snapin has a specific or primary function during SNARE-mediated synaptic vesicle fusion. Ultimately, genetic studies may be required to highlight the primary functions of this molecule. The recent generation of snapin mutant mice has demonstrated a dramatic effect on synchronized vesicle release (Tian et al., 2005; Pan et al., 2009) and endosomal transport (Cai et al., 2010). Neurodegeneration is also reported in these mutants (Cai et al., 2010).

In snapin mutant mice, evoked synaptic transmission at physiological calcium is decreased by $\sim 75 \%$, the synchrony of vesicle fusion is impaired, and there is a dramatic reduction in the rate of spontaneous vesicle fusion (Pan et al., 2009). These data clearly demonstrate that Snapin has a critical role in baseline neurotransmission. This function appears to be mediated through molecular interactions with both SNAP25 and Synaptotagmin1 (Ilardi et al., 1999; Krapivinsky et al., 2006; Pan et al., 2009). However, because baseline transmission is so severely perturbed in the mouse mutant, it is difficult to assess whether Snapin might also participate in various forms of neural plasticity beyond the short-term modulation of vesicle release during short trains of stimuli (Pan et al., 2009).

Our loss-of-function analysis of snapin in Drosophila highlights a unique function during the homeostatic modulation of neurotransmission. This function of Snapin may also be mediated through its interaction with SNAP25, based on the observation that animals that are doubly heterozygous for mutations in snapin and snap25 lack the expression of homeostatic plasticity without a major defect in baseline neurotransmission. An interesting possibility is that Snapin could impose homeostatic regulation on SNAREmediated fusion events via an interaction with SNAP25. This may, in fact, represent a common function of Snapin in other systems that are also under homeostatic control, including the regulation of calcium stores (Suzuki et al., 2007; Mohl et al., 2011) and ionic balance (Mistry et al., 2007, 2010; Yasuhara et al., 2008) in nonneural cells. However, based on our genetic interaction data, we cannot rule out the possibility that SNAP25 functions in parallel to Snapin during homeostatic plasticity.

Molecular mechanisms underlying the homeostatic control of presynaptic release at the neuromuscular junction

Recently, genetic studies have begun to identify genes that are necessary for the homeostatic control presynaptic neurotransmitter release at the Drosophila NMJ. In each example, a gene mutation prevents the homeostatic modulation of presynaptic release following perturbation of postsynaptic glutamate receptor function. Several mutations have been identified that disrupt baseline neurotransmission without altering the capacity to express synaptic homeostasis, including cystein string protein, methusela, and Hsc70 (Dickman and Davis, 2009). Thus, mutations that disrupt basal neurotransmission can be distinguished from those specifically involved in synaptic homeostasis by examining the effects of a mutation before and after disruption of glutamate receptor function. A growing list of genes fit these criteria for being involved in homeostatic plasticity within the presynaptic nerve terminal, including the cacophony (CaV2.1) calcium channel (Frank et al., 2006), EphR-ephexin-cdc42 signaling (Frank et al., 2009), dysbindin (Dickman and Davis, 2009), Rab3-GAP (Müller et al., 2011), gooseberry (Marie et al., 2010), the miR-310 group, and Khc-73 (Tsurudome et al., 2010). Here we add to this growing list by implicating snapin and possibly snap25.

A challenge for future studies will be to determine how the functions of these molecules are coordinated within a robust homeostatic signaling system capable of precisely tuning neurotransmission over a broad physiological range (Frank et al., 2006). It seems likely that the presynaptic calcium channel will be a focal point of this signaling system. However, some of these 
Table 1. Absolute values for normalized data

\begin{tabular}{|c|c|c|c|c|c|c|c|}
\hline Figure & Genotype & {$\left[\mathrm{Ca}^{2+}\right]$} & PhTX & mEJP & EJP & Quantal content (corrected) & $n$ \\
\hline $5 D$ & $w^{1118}$ & 0.5 & - & $0.95(0.02)$ & $35.41(0.80)$ & 71.48 (3.16) & 17 \\
\hline 50 & $w^{1118}$ & 0.5 & + & $0.52(0.03)$ & 34.20 (1.84) & $128.49(10.30)$ & 16 \\
\hline 50 & syt $t^{A D 4 /+}$ & 0.5 & - & $0.97(0.02)$ & $30.56(0.99)$ & $53.73(2.85)$ & 9 \\
\hline 50 & syt $\mathrm{t}^{A 4 /+}$ & 0.5 & + & $0.48(0.01)$ & $29.29(1.34)$ & $102.84(9.98)$ & 7 \\
\hline $5 D$ & Dysb $b^{1 /+}$ & 0.5 & - & $0.86(0.04)$ & $31.58(1.33)$ & $67.69(5.36)$ & 19 \\
\hline $5 D$ & Dysh $^{1 /+}$ & 0.5 & + & $0.40(0.03)$ & $27.99(2.17)$ & $127.57(12.34)$ & 20 \\
\hline 50 & syt $^{A D 4 /+} ; d y s b^{1 /+}$ & 0.5 & - & $0.89(0.05)$ & $28.36(1.25)$ & $53.02(3.55)$ & 11 \\
\hline $5 D$ & $s y t^{A D 4 /+} ; d y s b^{1 /+}$ & 0.5 & + & $0.46(0.20)$ & $26.00(0.76)$ & $86.81(3.81)$ & 8 \\
\hline $7 A$ & snapin $^{\text {DF(2L)Exel6277/+ }}$ & 0.5 & - & $0.94(0.05)$ & $31.58(1.33)$ & $55.31(4.17)$ & 10 \\
\hline $7 A$ & snapin $^{\text {DF(2L)Exel6277/+ }}$ & 0.5 & + & $0.49(0.02)$ & $28.33(0.65)$ & $55.31(4.17)$ & 9 \\
\hline $7 A$ & $D C^{1229 /+}$ & 0.5 & - & $0.97(0.04)$ & $29.80(1.24)$ & $51.43(2.14)$ & 10 \\
\hline $7 A$ & $D I C^{1229 /+}$ & 0.5 & + & $0.51(0.02)$ & $30.60(4.88)$ & $124.51(14.37)$ & 10 \\
\hline $7 A$ & dic $^{1229 /+} ;$ snapin $^{\text {DF(2L)Exel6277/+ }}$ & 0.5 & - & $0.92(0.05)$ & $29.43(1.38)$ & $53.14(2.25)$ & 7 \\
\hline $7 A$ & $\mathrm{dic}^{1229 /+} ;$; snapin DF(2L)Exel6277/+ & 0.5 & + & $0.55(0.03)$ & $28.80(1.08)$ & $90.66(10.71)$ & 10 \\
\hline $7 B$ & $\operatorname{snap} 25^{m \times 1 /+4}$ & 0.5 & - & $0.94(0.04)$ & $30.04(0.79)$ & $54.25(1.67)$ & 12 \\
\hline $7 B$ & snap $25^{m \times 124 /+}$ & 0.5 & + & $0.47(0.02)$ & $27.25(1.46)$ & $92.79(6.09)$ & 8 \\
\hline $7 B$ & $B \operatorname{los} 7^{E \times 2}$ & 0.5 & - & $0.95(0.04)$ & $29.00(1.03)$ & $30.53(1.79)$ & 6 \\
\hline $7 B$ & $B \operatorname{los} 7^{E \times 2}$ & 0.5 & + & $0.43(0.02)$ & $26.12(0.79)$ & $61.46(2.36)$ & 7 \\
\hline $7 B$ & snapin $^{\text {DF(2L)Exel6277/++ }}$; snap $25^{\text {mx124/+ }}$ & 0.5 & - & $0.96(0.03)$ & $27.33(1.54)$ & $46.30(3.68)$ & 12 \\
\hline $7 B$ & $\begin{array}{l}\text { snapin }^{\text {DF(2L)Exel6277/+ }} \text {; snap 25 } 5^{\text {m12124/+ }} \\
\text { snapin }^{\text {DF(2L)Exel6277/+ }} / 0 K 371-G A L 4\end{array}$ & 0.5 & + & $0.45(0.02)$ & $15.73(1.26)$ & $45.34(4.61)$ & 11 \\
\hline $7 B$ & $\begin{array}{l}\text { snap25 }{ }^{\text {mx124/+ }} / \text { UAS-snapin } \\
\text { snapin }^{\text {DF(2L)Exel6277/+ } / O K 371-G A L 4}\end{array}$ & 0.5 & - & $0.92(0.02)$ & $30.46(1.13)$ & $56.92(4.23)$ & 11 \\
\hline $7 B$ & snap $25^{m \times 124 /+} /$ UAS-snapin & 0.5 & + & $0.47(0.01)$ & $25.50(0.98)$ & $83.14(4.33)$ & 8 \\
\hline
\end{tabular}

The figure and panel, genotype, and conditions used are noted. In addition, we indicate external calcium concentration and whether or not PhTx was applied. Average values for mEJP, EJP, $Q \mathrm{C}$, and number of data samples ( $n$ ) are shown with SE in parentheses.

\section{A}

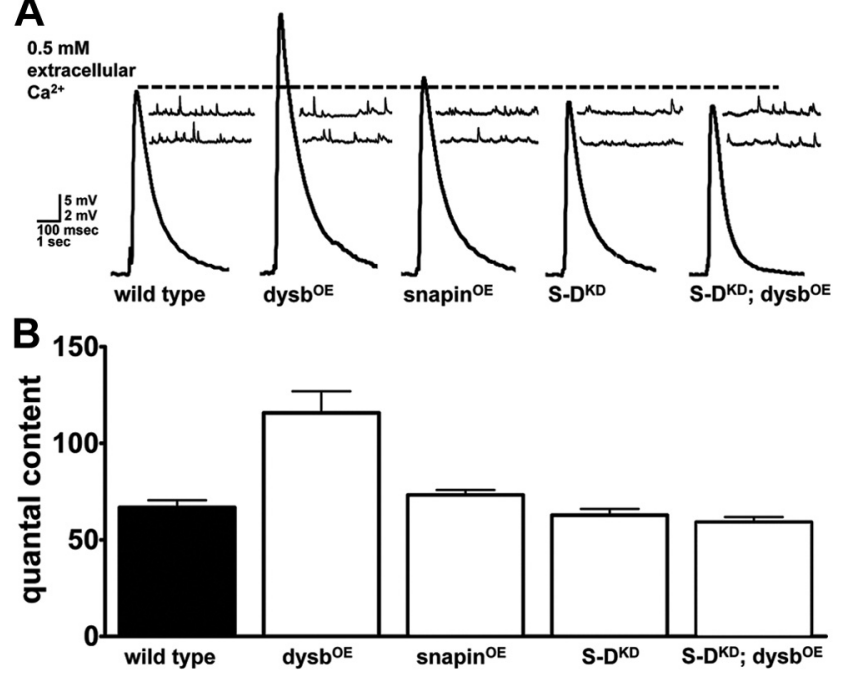

Figure 6. Potentiation of release by dysbindin requires snapin. $A$, Representative traces of baseline evoked EJP amplitudes in wild-type $(w ;+;+; n=16)$, dysbindin overexpression (c155-GAL4/Y;+;UAS-dysb/+;n=17), snapin overexpression (c155-GAL4/Y;UAS-snapin/ $+; n=$

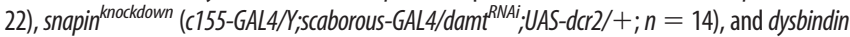
overexpression in a snapin ${ }^{\text {knockdown }}$ mutant background (C155-GAL4/Y; scaborous-GAL4/damt ${ }^{\text {RNAi; }}$; UAS-dcr2/UAS-dysb; $n=14)$. B, Quantal contents of indicated genotypes. Error bars are SEM.

molecules may identify additional layers of modulation including dysbindin, snapin, and snap25. Furthermore, miR-310 and $K h c-73$ have been proposed to regulate active zone structure, which could have a direct effect on calcium channels or other parameters that modulate vesicle release. A further challenge will be to determine not only whether or not these molecules are necessary, but how they normally participate in the induction and expression of a homeostatic change in presynaptic release. Ultimately, these molecular studies will need to be combined with our physiological understanding of this process (Weyhers-

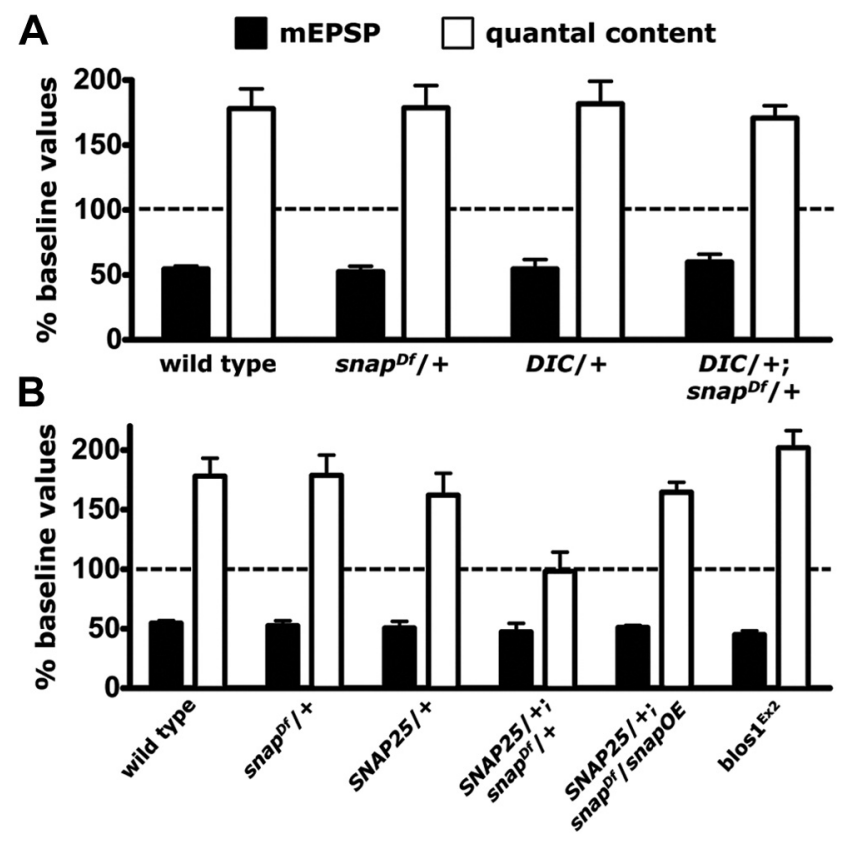

Figure 7. snapin genetically interacts with snap25 but not dynein intermediate chain during synaptichomeostasis. $\boldsymbol{A}$, No genetic interaction is observed between a snapin deficiency (Df(2L)Exel6277) and a strong loss-of-function allele of dynein intermediate chain $\left(\mathrm{dic}^{1229}\right)$ when the NMJ is challenged following PhTx application ( $p>0.05$, Student's $t$ test and ANOVA). $\boldsymbol{B}$, Strong genetic interaction between the snapin deficiency and a null mutation in snap25 $\left(\operatorname{snap} 25^{m \times 124}\right)$, where there is no increase in quantal content following PhTx application ( $p<0.0037$, Student's $t$ test and ANOVA). Synaptic homeostasis can be restored when snapin is expressed in motorneurons (c155-GAL4/Y. snap $25^{m \times 124} /$ snapin $^{D F(2 L) E x e l 6277}$; UAS-snapin/+). Synaptic homeostasis is robustly expressed in mutants in the Bloc-1 subunit blos 1 ( $w$; blos $7^{\text {ex2 }}$ ). Error bars are SEM.

müller et al., 2011) and with new imaging approaches to visualize the dynamic molecular interactions in vivo that drive homeostatic plasticity. 


\section{References}

Bergquist S, Dickman DK, Davis GW (2010) A hierarchy of cell intrinsic and target-derived homeostatic signaling. Neuron 66:220-234.

Buxton P, Zhang XM, Walsh B, Sriratana A, Schenberg I, Manickam E, Rowe $\mathrm{T}$ (2003) Identification and characterization of Snapin as a ubiquitously expressed SNARE-binding protein that interacts with SNAP23 in nonneuronal cells. Biochem J 375:433-440.

Cai Q, Lu L, Tian JH, Zhu YB, Qiao H, Sheng ZH (2010) Snapin-regulated late endosomal transport is critical for efficient autophagy-lysosomal function in neurons. Neuron 68:73-86.

Cheli VT, Dell'Angelica EC (2010) Early origin of genes encoding subunits of biogenesis of lysosome-related organelles complex-1, -2 and -3 . Traffic 11:579-586.

Cheli VT, Daniels RW, Godoy R, Hoyle DJ, Kandachar V, Starcevic M, Martinez-Agosto JA, Poole S, DiAntonio A, Lloyd VK, Chang HC, Krantz DE, Dell'Angelica EC (2010) Genetic modifiers of abnormal organelle biogenesis in a Drosophila model of BLOC-1 deficiency. Hum Mol Genet 19:861-878.

Davis GW (2006) Homeostatic control of neural activity: from phenomenology to molecular design. Annu Rev Neurosci 29:307-323.

Davis GW, Goodman CS (1998) Genetic analysis of synaptic development and plasticity: homeostatic regulation of synaptic efficacy. Curr Opin Neurobiol 8:149-156.

DiAntonio A, Parfitt KD, Schwarz TL (1993) Synaptic transmission persists in synaptotagmin mutants of Drosophila. Cell 73:1281-1290.

Dickman DK, Davis GW (2009) The schizophrenia susceptibility gene dysbindin controls synaptic homeostasis. Science 326:1127-1130.

Falcón-Pérez JM, Starcevic M, Gautam R, Dell'Angelica EC (2002) BLOC-1, a novel complex containing the pallidin and muted proteins involved in the biogenesis of melanosomes and platelet-dense granules. J Biol Chem 277:28191-28199.

Frank CA, Kennedy MJ, Goold CP, Marek KW, Davis GW (2006) Mechanisms underlying the rapid induction and sustained expression of synaptic homeostasis. Neuron 52:663-677.

Frank CA, Pielage J, Davis GW (2009) A presynaptic homeostatic signaling system composed of the Eph receptor, ephexin, Cdc42, and CaV2.1 calcium channels. Neuron 61:556-569.

Ghiani CA, Dell'angelica EC (2011) Dysbindin-containing complexes and their proposed functions in brain: from zero to (too) many in a decade. ASN Neuro 3:pii:e00058.

Ilardi JM, Mochida S, Sheng ZH (1999) Snapin: a SNARE-associated protein implicated in synaptic transmission. Nat Neurosci 2:119-124.

Jakawich SK, Nasser HB, Strong MJ, McCartney AJ, Perez AS, Rakesh N, Carruthers CJ, Sutton MA (2010) Local presynaptic activity gates homeostatic changes in presynaptic function driven by dendritic BDNF synthesis. Neuron 68:1143-1158.

Kim SH, Ryan TA (2010) CDK5 serves as a major control point in neurotransmitter release. Neuron 67:797-809.

Kittel RJ, Wichmann C, Rasse TM, Fouquet W, Schmidt M, Schmid A, Wagh DA, Pawlu C, Kellner RR, Willig KI, Hell SW, Buchner E, Heckmann M, Sigrist SJ (2006) Bruchpilot promotes active zone assembly, Ca2+ channel clustering, and vesicle release. Science 312:1051-1054.

Krapivinsky G, Mochida S, Krapivinsky L, Cibulsky SM, Clapham DE (2006) The TRPM7 ion channel functions in cholinergic synaptic vesicles and affects transmitter release. Neuron 52:485-496.

Littleton JT, Stern M, Schulze K, Perin M, Bellen HJ (1993) Mutational analysis of Drosophila synaptotagmin demonstrates its essential role in $\mathrm{Ca}(2+)$-activated neurotransmitter release. Cell 74:1125-1134.

Lu L, Cai Q, Tian JH, Sheng ZH (2009) Snapin associates with late endocytic compartments and interacts with late endosomal SNAREs. Biosci Rep 29:261-269.

Marder E, Goaillard JM (2006) Variability, compensation and homeostasis in neuron and network function. Nat Rev Neurosci 7:563-574.

Marie B, Pym E, Bergquist S, Davis GW (2010) Synaptic homeostasis is consolidated by the cell fate gene gooseberry, a Drosophila pax3/7 homolog. J Neurosci 30:8071-8082.

Marrus SB, Portman SL, Allen MJ, Moffat KG, DiAntonio A (2004) Differential localization of glutamate receptor subunits at the Drosophila neuromuscular junction. J Neurosci 24:1406-1415.

Martin AR (1955) A further study of the statistical composition on the endplate potential. J Physiol 130:114-122.

Mistry AC, Mallick R, Fröhlich O, Klein JD, Rehm A, Chen G, Sands JM
(2007) The UT-A1 urea transporter interacts with snapin, a SNAREassociated protein. J Biol Chem 282:30097-30106.

Mistry AC, Mallick R, Klein JD, Sands JM, Fröhlich O (2010) Functional characterization of the central hydrophilic linker region of the urea transporter UT-A1: cAMP activation and snapin binding. Am J Physiol Cell Physiol 298:C1431-C1437.

Mohl MC, Iismaa SE, Xiao XH, Friedrich O, Wagner S, Nikolova-Krstevski V, Wu J, Yu ZY, Feneley M, Fatkin D, Allen DG, Graham RM (2011) Regulation of murine cardiac contractility by activation of alpha(1A)adrenergic receptor-operated $\mathrm{Ca}(2+)$ entry. Cardiovasc Res 91:310-319.

Müller M, Pym EC, Tong A, Davis GW (2011) Rab3-GAP controls the progression of synaptic homeostasis at a late stage of vesicle release. Neuron 69:749-762.

Numakawa T, Yagasaki Y, Ishimoto T, Okada T, Suzuki T, Iwata N, Ozaki N, Taguchi T, Tatsumi M, Kamijima K, Straub RE, Weinberger DR, Kunugi H, Hashimoto R (2004) Evidence of novel neuronal functions of dysbindin, a susceptibility gene for schizophrenia. Hum Mol Genet 13:2699-2708.

Pan PY, Tian JH, Sheng ZH (2009) Snapin facilitates the synchronization of synaptic vesicle fusion. Neuron 61:412-424.

Petersen SA, Fetter RD, Noordermeer JN, Goodman CS, DiAntonio A (1997) Genetic analysis of glutamate receptors in Drosophila reveals a retrograde signal regulating presynaptic transmitter release. Neuron 19:1237-1248.

Pielage J, Fetter RD, Davis GW (2006) A postsynaptic spectrin scaffold defines active zone size, spacing, and efficacy at the Drosophila neuromuscular junction. J Cell Biol 175:491-503.

Pozo K, Goda Y (2010) Unraveling mechanisms of homeostatic synaptic plasticity. Neuron 66:337-351.

Qin G, Schwarz T, Kittel RJ, Schmid A, Rasse TM, Kappei D, Ponimaskin E, Heckmann M, Sigrist SJ (2005) Four different subunits are essential for expressing the synaptic glutamate receptor at neuromuscular junctions of Drosophila. J Neurosci 25:3209-3218.

Suzuki F, Morishima S, Tanaka T, Muramatsu I (2007) Snapin, a new regulator of receptor signaling, augments alphalA-adrenoceptor-operated calcium influx through TRPC6. J Biol Chem 282:29563-29573.

Talbot K, Cho DS, Ong WY, Benson MA, Han LY, Kazi HA, Kamins J, Hahn CG, Blake DJ, Arnold SE (2006) Dysbindin-1 is a synaptic and microtubular protein that binds brain snapin. Hum Mol Genet 15:3041-3054.

Tian JH, Wu ZX, Unzicker M, Lu L, Cai Q, Li C, Schirra C, Matti U, Stevens D, Deng C, Rettig J, Sheng ZH (2005) The role of Snapin in neurosecretion: snapin knock-out mice exhibit impaired calcium-dependent exocytosis of large dense-core vesicles in chromaffin cells. J Neurosci 25:10546-10555.

Tsurudome K, Tsang K, Liao EH, Ball R, Penney J, Yang JS, Elazzouzi F, He T, Chishti A, Lnenicka G, Lai EC, Haghighi AP (2010) The Drosophila miR-310 cluster negatively regulates synaptic strength at the neuromuscular junction. Neuron 68:879-893.

Turrigiano G (2011) Too many cooks? Intrinsic and synaptic homeostatic mechanisms in cortical circuit refinement. Annu Rev Neurosci 34:89-103.

Turrigiano GG (2008) The self-tuning neuron: synaptic scaling of excitatory synapses. Cell 135:422-435.

Turrigiano GG, Nelson SB (2004) Homeostatic plasticity in the developing nervous system. Nat Rev Neurosci 5:97-107.

Vilinsky I, Stewart BA, Drummond J, Robinson I, Deitcher DL (2002) A Drosophila SNAP-25 null mutant reveals context-dependent redundancy with SNAP-24 in neurotransmission. Genetics 162:259-271.

Vites O, Rhee JS, Schwarz M, Rosenmund C, Jahn R (2004) Reinvestigation of the role of snapin in neurotransmitter release. J Biol Chem 279:26251-26256.

Wall AA, Phillips AM, Kelly LE (2005) Effective translation of the second cistron in two Drosophila dicistronic transcripts is determined by the absence of in-frame AUG codons in the first cistron. J Biol Chem 280:27670-27678.

Weyhersmüller A, Hallermann S, Wagner N, Eilers J (2011) Rapid active zone remodeling during synaptic plasticity. J Neurosci 31:6041-6052.

Yasuhara A, Wada J, Malakauskas SM, Zhang Y, Eguchi J, Nakatsuka A, Murakami K, Kanzaki M, Teshigawara S, Yamagata K, Le TH, Makino H (2008) Collectrin is involved in the development of salt-sensitive hypertension by facilitating the membrane trafficking of apical membrane proteins via interaction with soluble $\mathrm{N}$-ethylmaleiamide-sensitive factor attachment protein receptor complex. Circulation 118:2146-2155.

Zheng Y, Wildonger J, Ye B, Zhang Y, Kita A, Younger SH, Zimmerman S, Jan LY, Jan YN (2008) Dynein is required for polarized dendritic transport and uniform microtubule orientation in axons. Nat Cell Biol 10:1172-1180. 